\title{
A molecular dynamics simulation to investigate the thermal properties of SWCNT/poly(phenylenesulfone) nanocomposites
}

\author{
Siavash Taheri • Muhammad Shadman • \\ Zohreh Ahadi · Farid Asgari · Hossein Mighani
}

Received: 18 March 2014/ Accepted: 13 June 2014/Published online: 25 July 2014

(c) The Author(s) 2014. This article is published with open access at Springerlink.com

\begin{abstract}
An equilibrium molecular dynamics simulation is applied to investigate the thermal properties of a singlewalled carbon nanotube/poly(phenylenesulfone) as nanocomposite material. Cohesive energy density and the Hildebrand solubility parameter of pure poly (phenylenesulfone) and nanocomposite are calculated to compare the thermal analysis of them. The results indicate that carbon nanotube/poly(phenylenesulfone) nanocomposites are thermally stable than pure poly(phenylenesulfone); however, poly(phenylenesulfone) is a thermally stable polymer. This means carbon nanotube can further improve thermal properties of thermally stable polymer.
\end{abstract}

Keywords Nanocomposites - Carbon nanotube ·

Molecular dynamics · Phenylenesulfone · Cohesive energy density

\section{Introduction}

Molecular dynamics (MD) simulation is one of the powerful tools to investigate chemical and physical structural and dynamical property details of various types of materials such as polymers at the atomic level and to link these

S. Taheri · Z. Ahadi · F. Asgari

Department of Science and Engineering, Abhar Branch, Islamic Azad University, Abhar, Iran

M. Shadman $(\square)$

Department of Chemistry, Faculty of Science, University of Zanjan, P.O. Box 45195-313, Zanjan, Iran

e-mail: shadman@znu.ac.ir

H. Mighani

Department of Chemistry, Golestan University, Gorgan, Iran simulation observations to experimentally accessible macroscopic properties of polymers [1]. Recently, nanotubes/polymer nanocomposites have been considered more attention, because their noticeable thermal and mechanical properties of them than pure polymer those nanotubes/ polymer nanocomposites represent with regard to conventional polymer compositions [2].

Since the discovery of carbon nanotubes (CNTs) in 1991 by Iijima [3], it has found wonderful industrial, scientific and engineering communities' interests of them. This is due to their specific properties such as thermal, mechanical and electronic behavior and remarkable structural; the CNTs are attended as invaluable reinforcements for improvement of chemical and physical structural and multi-functional composite properties $[4,5]$.

Poly(phenylenesulfone) has one of the most prominent applications. It is as constituent of proton exchange membrane (PEM) fuel cell high temperatures $\left(\mathrm{T}<130{ }^{\circ} \mathrm{C}\right)$ in a humid environment [6]. Also, it does not depict a glass transition temperature $\left(\mathrm{T}_{\mathrm{g}}\right)$ due to its very rigid backbone and it remains in a glassy state even at very high temperatures $[6,7]$. In Fig. 1, we can find units $\left(-\mathrm{SO}_{2}-\right)$ connecting the phenyl rings in poly(phenylenesulfone) that this functional group causes the thermal properties can be achieved.

Therefore, with most presumably, at high temperatures, the glassy poly(phenylenesulfone) exhibits a rich behavior under deformation. In this case, using MD as a computational method and as very sufficient popular tool, we can model a nanocomposite consisting of a single-walled carbon nanotube (SWCNT) embedded in poly(phenylenesulfone) matrix. Applying MD, this model can predict the chemical and physical properties of achieved nanocomposite at high temperatures. This method helps us to obtain a new composition to extend the use of low-cost materials. In fact, by distinguishing the trajectories' atoms in this 
Fig. 1 The structure of phenylenesulfone used in this work. $\mathrm{C}, \mathrm{H}, \mathrm{O}$ and $\mathrm{S}$ are gray, white, red and yellow atoms nanocomposite using integrating Newton's second law of motion, we can determine the mechanical, thermal and other properties of SWCNT/poly(phenylenesulfone).

In this paper, at first, we describe a MD procedure to produce poly (phenylenesulfone) model. Also, SWCNT/ poly (phenylenesulfone) nanocomposite generation with MD technique explains in computational details and in the last section, we present the obtained data and exhibit the results and discussion.

\section{MD computational details}

Pure poly(phenylenesulfone)

In the first stage, a chain of phenylenesulfone consisting four monomers has been considered, (the monomer is represented in Fig. 1) and they must be minimized with a equilibrium molecular mechanics (EMD) approach and then using the global update method referred to as the Cartesian Hybrid Monte Carlo (CHMC) algorithm [8], 22 chains of phenylenesulfone have been generated as a sequence of configurations of the bulk poly(phenylenesulfone) with probabilities appropriate for the NVT ensemble (Canonical Ensemble). It should be noted that, the obtained density of dry poly(phenylenesulfone) in our simulation is $1.66 \mathrm{~g} \mathrm{~cm}^{-3}$; while it is corresponded approximately which completely disordered amorphous

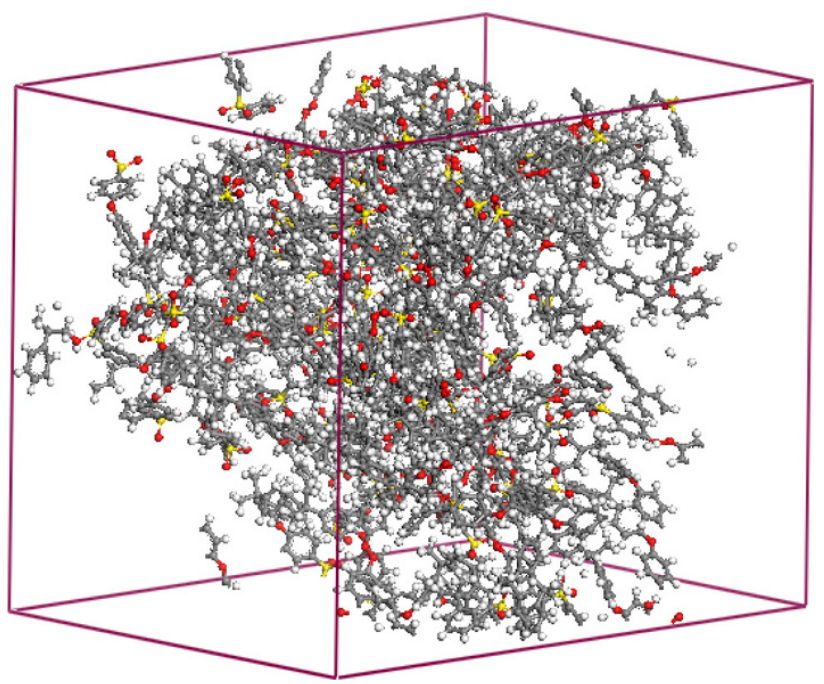

Fig. 2 The PBC structure of poly(phenylenesulfone) with 4,796 atoms

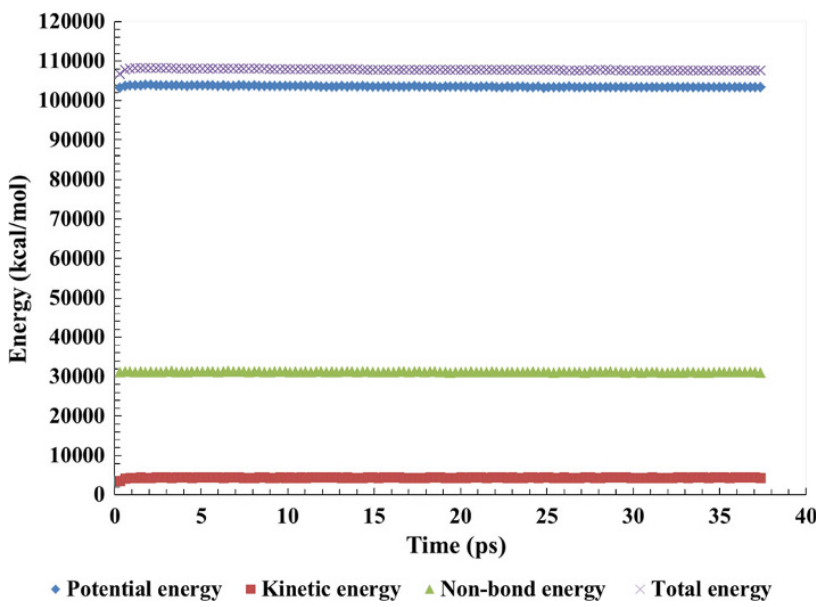

Fig. 3 The energies of poly(phenylenesulfone) simulation cell after equilibration step

solid phase poly(phenylenesulfone) [6]. To run next MD steps' simulation of poly(phenylenesulfone), it is necessary to apply the three-dimensional boundary condition (PBC) on the structure of this polymer. (the PBC structure of poly(phenylenesulfone) is shown in Fig. 2).

In continuation of the simulation, a NPT ensemble is applied on simulation cell, to achieve equilibrium at energy and temperature. Figures 3 and 4 show the energies and temperature of poly(phenylenesulfone) simulation cell after equilibration step, respectively. Furthermore, these Figures illustrate energy and temperature after $10 \mathrm{ps}$ that poly(phenylenesulfone) simulation cell is equilibrated and it is ready to the production step of MD simulation. 


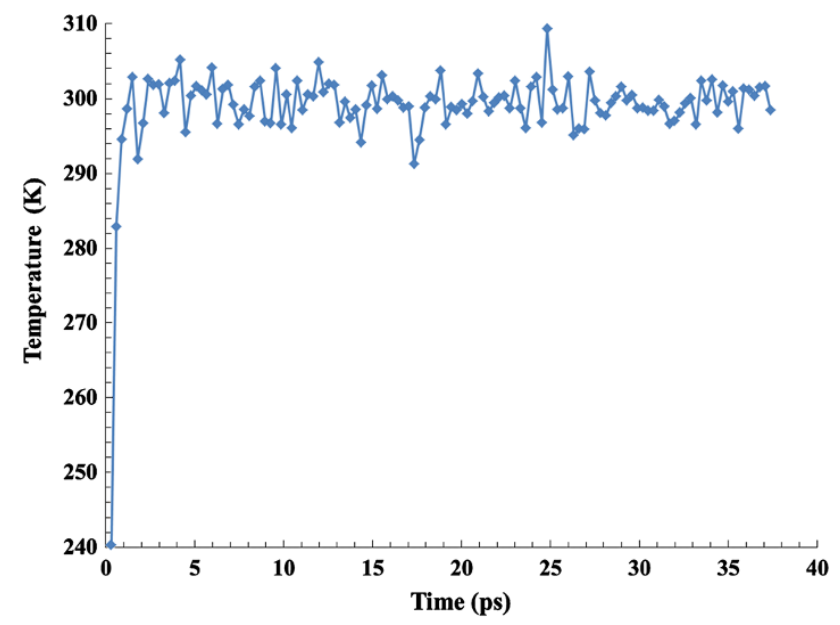

Fig. 4 The temperature of poly(phenylenesulfone) simulation cell after equilibration step

Moreover, it should be noted that in all simulations, the DREIDING force field [9] with 1 fs time step is applied. Also, the standard Ewald summation technique [10] is used to calculate the effect of the non-bond electrostatic energy of the PBC structure of poly(phenylenesulfone) and The DLPOLY package is used to all calculations [11].

After equilibration step on simulation cell and achieve the minimum energy of composition of matrix, we performed an anneal procedure with molecular dynamics which it is included some consecutive NPT dynamics simulation cycles with temperature treatment. Each of these short NPT simulations in each cycle have several tens ps simulation length separately, while the temperature in each cycle is periodically increased from 250 to $650 \mathrm{~K}$ and then decreased again to finish a cycle. Furthermore, in whole of these steps, the simulation length for each cycle is $5.0 \mathrm{~ns}$. With anneal procedure, we will be generated the lowest energy on composition of matrix without trapping the structure. In fact, an anneal method with applying increasing temperature and then decreases can cause thermodynamics stability structure on polymer or composite. Then, we can receive the response of polymer to this temperature treatment.

As a result, Fig. 5 presents one of the initial cycle of anneal molecular dynamics of poly(phenylenesulfone) simulation cell. This has 80 ps simulation length and the following annealing processes have a much longer simulation time.

\section{SWCNT/poly(phenylenesulfone) nanocomposite}

Therefore, here, for the preparation of SWCNT/ poly(phenylenesulfone) in this present work, we have selected a $(10,10)$ SWCNT with $100 \AA$ tube length and 22 chains of phenylenesulfone which were placed around the

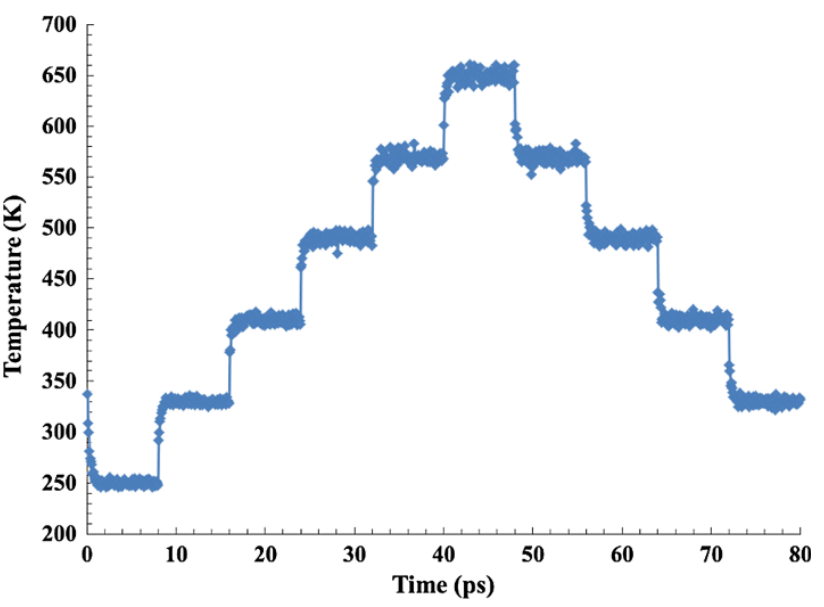

Fig. 5 The initial anneal molecular dynamics of poly(phenylenesulfone) simulation cell

nanotube (Fig. 6a). Then, molecular geometry optimization and molecular dynamics are calculated at ambient temperature on new nanocomposite to reach equilibrium energetically. Thus, the cross-section structure of SWCNT/ poly(phenylenesulfone) has shown in Fig. 6b. The simulation length in this step was $5.0 \mathrm{~ns}$, which is equal to simulation time for pure poly(phenylenesulfone) under annealing process.

However, after MD equilibration step on $(10,10)$ SWCNT/poly(phenylenesulfone), the annealing dynamics must be carried out to allow the energy of this nanocomposite to be minimized gradually without trapping the chemical structure in a conformation that represents a minimum local energy. Also, the lower and higher temperature here are the same in annealing step of pure poly(phenylenesulfone). Figure 7 shows the last cycle of annealing process of SWCNT/poly(phenylenesulfone). Therefore, both pure and nanocomposite of poly(phenylenesulfone) have the same anneal dynamics process with an equal number of thermal cycles and simulation length. This helps us to compare the thermal and mechanical properties of both of pure and nanocomposite of poly(phenylenesulfone) at same thermodynamics conditions. We have only investigated the thermal properties of them and the mechanical properties will be reported in another work.

\section{Results and discussion}

Cohesive energy density

To indicate the thermal properties of each material, the cohesive energy (CE) and cohesive energy density (CED) must be determined [12]. Here, briefly, we describe CED 

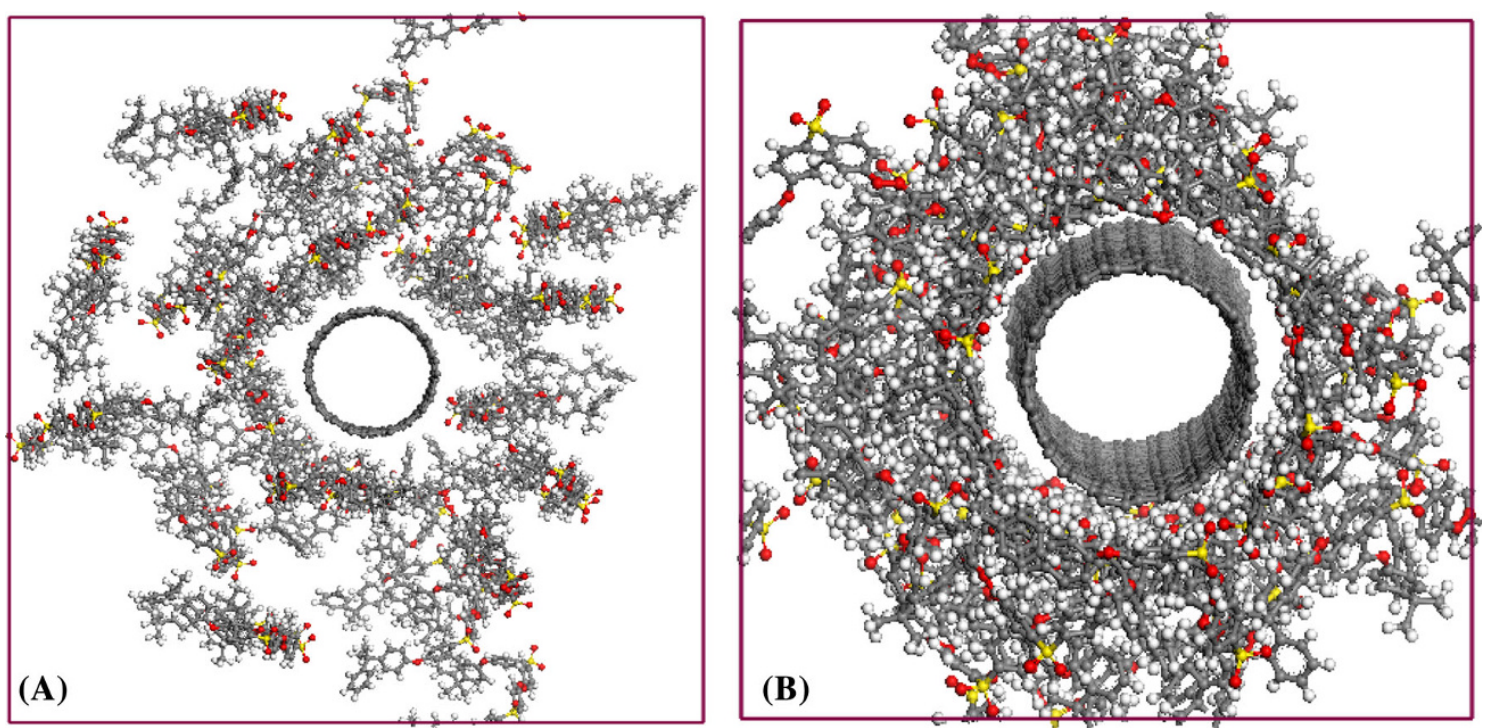

Fig. 6 a The cross-section of initial structure of SWCNT/poly(phenylenesulfone). b The cross-section structure of SWCNT/poly(phenylenesulfone) after MD equilibration step

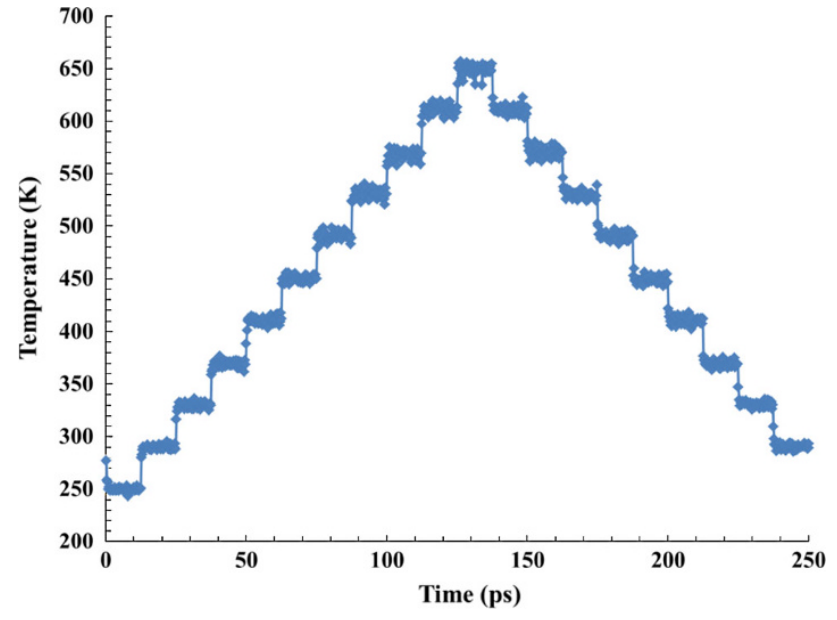

Fig. 7 The last cycle of anneal dynamics of SWCNT/ poly(phenylenesulfone)

calculation from MD simulation. In atomistic molecular dynamics simulations, the $\mathrm{CE}$ is the amount of energy needed to completely remove unit volume of molecules from their neighbors to infinite separation-which it means all intermolecular forces are eliminated-so it is equal to the heat of vaporization divided by molar volume. Therefore, the CED corresponds to the cohesive energy per unit volume.

Hildebrand solubility parameter

The Hildebrand solubility parameter $\left(\delta_{H}\right)$ is the square root of the CED: $\delta_{\mathrm{H}}=\mathrm{CED}^{0.5}=(\Delta U / V)^{0.5}=\left[\left(\Delta H^{\mathrm{vap}}-R T\right) /\right.$

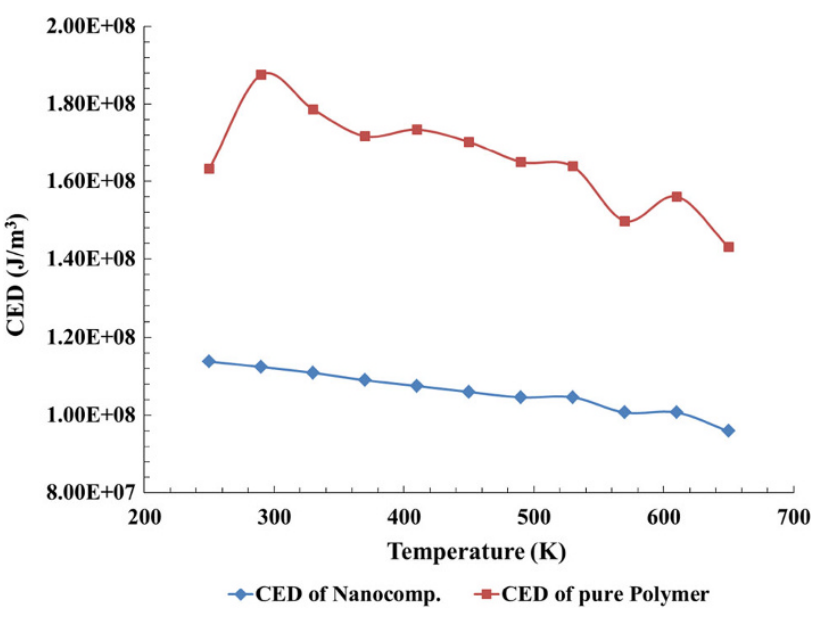

Fig. 8 The CED of pure and nanocomposite of poly(phenylenesulfone)

$V]^{0.5}$, where $\Delta U, \Delta H^{\mathrm{vap}}$ and $V$ are the molar internal energy, the enthalpy of vaporization (at $298 \mathrm{~K}$ ) and the molar volume, respectively [12].

After the annealing dynamics, the cohesive energy density (CED) and the Hildebrand solubility parameter are calculated for each temperature of the last cycle of anneal process in both pure and nanocomposite of poly(phenylenesulfone). Figures 8 and 9 show the CED and $\delta_{\mathrm{H}}$ of pure and nanocomposite of poly(phenylenesulfone), respectively. Figure 8 shows that the CED of both pure and nanocomposite of poly(phenylenesulfone) decrease as increasing of temperature while the CED of nanocomposite is lower than the CED of pure poly(phenylenesulfone). 


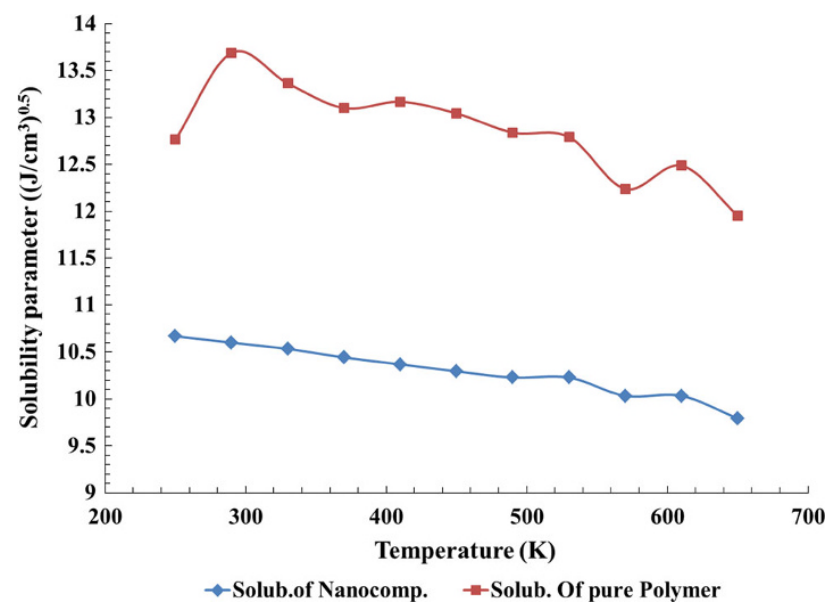

Fig. $9 \delta_{\mathrm{H}}$ of pure and nanocomposite of poly(phenylenesulfone)

Also, Fig. 9 shows that the Hildebrand solubility parameter of both pure and nanocomposite of poly(phenylenesulfone) are a decreasing function of temperature while the Hildebrand solubility parameter of nanocomposite is lower than the Hildebrand solubility parameter of pure poly(phenylenesulfone). In Figs. 8 and 9, standard deviation error is lower than $3 \%$. According to these Figures, we can find that in nanocomposite, the CED and the Hildebrand solubility parameter is lower than the pure polymer. Consequently, decreasing of the CED and $\delta_{\mathrm{H}}$ can increase specific volume of nanocomposite more than pure polymer. This means $T_{g}$ of nanocomposite will be greater than pure polymer. However, this must be proved with applying non-equilibrium molecular dynamics procedure.

\section{Conclusion}

In this work, we report an EMD technique to study thermal stability of SWCNT/poly(phenylenesulfone). Although, poly(phenylenesulfone) is an well-known thermal stable polymer, our investigation is shown that SWCNT/ poly(phenylenesulfone) has more thermal stability than pure poly(phenylenesulfone). To this indication, the CED and Hildebrand solubility parameter of both pure poly(phenylenesulfone) and SWCNT/poly(phenylenesulfone) is calculated and the obtained results illustrate that the CED and the Hildebrand solubility parameter of SWCNT/ poly(phenylenesulfone) is lower than these parameters of pure poly(phenylenesulfone) polymer. The lower of CED and the Hildebrand solubility parameter show that specific volume of SWCNT/poly(phenylenesulfone) is more than pure poly(phenylenesulfone). Therefore, $\mathrm{T}_{\mathrm{g}}$ of SWCNT/ poly(phenylenesulfone) is higher than pure poly(phenylenesulfone).

Authors' contribution MS was involved in designing the simulation work and ZA wrote the program and ST and FA were involved in getting the data and drafting the manuscript. MS revised the manuscript critically and ZA and HM gave final approval for submission. All authors read and approved the final manuscript.

Acknowledgments We acknowledge the University of Zanjan and the Abhar branch of the Islamic Azad University Research Councils for support of this work.

Conflict of interest The authors declare that they have no competing interests.

Open Access This article is distributed under the terms of the Creative Commons Attribution License which permits any use, distribution, and reproduction in any medium, provided the original author(s) and the source are credited.

\section{References}

1. Fukui, K., Sumpter, B.G., Barnes, M.D., Noid, D.W.: Molecular dynamics studies of the structure and properties of polymer nanoparticles. Comput. Theor. Polym. Sci. 9, 245 (1999)

2. Zhang, J., Lou, J., Ilias, S., Krishnamachari, P., Yan, J.: Thermal properties of poly(lactic acid) fumed silica nanocomposites: experiments and molecular dynamics simulations. Polymer 49, $2381(2008)$

3. Iijima, S.: Helical microtubules of graphitic carbon. Nature 354, 56 (1991)

4. Chowdhury, S.C., Okabe, T.: Computer simulation of carbon nanotube pull-out from polymer by the molecular dynamics method. Compos. A 38, 747 (2007)

5. Han, Y., Elliott, J.: Molecular dynamics simulations of the elastic properties of polymer/carbon nanotube composites. Comput. Mater. Sci. 39, 315 (2007)

6. de Schuster, M., Araujo, C.C., Atanasov, V., Andersen, H.T., Kreuer, K.-D., Maier, J.: Highly sulfonated poly(phenylene sulfone): preparation and stability issues. Macromolecules 42, 3129 (2009)

7. Robello, D.R., Ulman, A., Urankar, E.J.: Poly(p-phenylene sulfone). Macromolecules 26, 6718 (1993)

8. Creutz, M., Gocksch, A.: Higher order hybrid Monte Carlo algorithms. Phys. Rev. Lett. 63, 9 (1989)

9. Mayo, S.L., Olafson III, B.D., Goddard, W.A.: DREIDING: a generic force field for molecular simulations. J. Phys. Chem. 94, 8897 (1990)

10. Ewald, P.P.: Die Berchnung optischer und elektrostatischer Gitterpotentiale. Ann. Phys. 64, 253 (1921)

11. DLPOLY code is obtained from Daresbury laboratory, UK

12. Hildebrand, J.H., Wood, S.E.: The derivation of equations for regular solutions. J. Chem. Phys. 1, 817 (1933) 